\title{
Subthalamic Nucleus Deep Brain Stimulation: An Invaluable Role for MER
}

\author{
F.A. Zeiler, M. Wilkinson, J.P. Krcek
}

\begin{abstract}
Introduction: Subthalamic nucleus (STN) deep brain stimulation (DBS) is currently the main surgical procedure for medically refractory Parkinson's disease. The benefit of intra-operative microelectrode recording (MER) for the purpose of neurophysiological localization and mapping of the STN continues to be debated. Methods: A retrospective review of the charts and operative reports of all patients receiving STN DBS implantation for Parkinson's disease at our institution from January 2004 to March 2011 was done. Results: Data from 43 of 44 patients with Parkinson's disease treated with STN DBS were reviewed. The average number of tracts on the left was 2.4 , versus 2.3 on the right. The average dorsal and ventral anatomical boundaries of the STN based on Schaltenbrand's Stereotactic Atlas were estimated to be at $-5.0 \mathrm{~mm}$ above and $+1.4 \mathrm{~mm}$ below target respectively. The average dorsal and ventral boundaries of the STN using MER were $-2.6 \mathrm{~mm}$ above and $+2.0 \mathrm{~mm}$ below target respectively. The average dorsal-ventral distance of the STN as predicted by Stereotactic Atlas was $6.4 \mathrm{~mm}$, compared to $4.6 \mathrm{~mm}$ as determined by MER. MER demonstrated the average dorsal and ventral boundaries on the left side were $-2.6 \mathrm{~mm}$ and $+2.2 \mathrm{~mm}$ from target respectively, while the average dorsal and ventral boundaries on the right side were $-2.5 \mathrm{~mm}$ and $+1.8 \mathrm{~mm}$ from target respectively with MER. Conclusions: MER in STN DBS surgery demonstrated measurable difference between stereotactic atlas/MRI STN target and neurophysiologic STN localization.
\end{abstract}

RÉSUMÉ: Stimulation cérébrale profonde du corps de Luys : rôle inestimable du système MER d'enregistrement extracellulaire par microélectrode. Contexte : La stimulation cérébrale profonde (SCP) du corps de Luys (CL) est actuellement la principale intervention chirurgicale utilisée dans le traitement de la maladie de Parkinson réfractaire au traitement médical. Le bénéfice de l'enregistrement par microélectrode (MER) pendant la chirurgie pour la localisation neurophysiologique et la cartographie du CL demeure un sujet de controverse. Méthode : Nous avons effectué une revue rétrospective des dossiers et des comptes rendus chirurgicaux de tous les patients atteints de la maladie de Parkinson qui ont reçu une implantation d'électrode pour la SCP du CL dans notre institution de janvier 2004 à mars 2011. Résultats : Les données de 43 des 44 patients atteints de la maladie de Parkinson traités par SCP du CL ont été revues. Le nombre moyen de faisceaux était 2,4 du côté gauche et 2,3 du côté droit. Les limites anatomiques dorsales et ventrales moyennes du CL selon l'atlas d'anatomie stéréotaxique de Schaltenbrand se situaient à $-5,0 \mathrm{~mm}$ au dessus et $+1,4$ mm sous la cible respectivement selon notre estimé. Les limites dorsales et ventrales moyennes du CL en utilisant le MER étaient -2,6 mm au dessus et $+2,0$ $\mathrm{mm}$ sous la cible respectivement. La distance dorsale-ventrale moyenne du CL prédite au moyen de l'atlas était 6,4 mm comparée à 4,6 mm selon la détermination par le MER. Le MER a montré que les limites dorsales et ventrales moyennes du côté gauche étaient à $-2,6 \mathrm{~mm}$ et $+2,2 \mathrm{~mm}$ de la cible respectivement, alors que les limites dorsales et ventrales moyennes du côté droit étaient à $-2,5 \mathrm{~mm}$ et $+1,8 \mathrm{~mm}$ de la cible respectivement avec le MER. Conclusions : Le MER dans chirurgie de la SCP du CL a montré une différence mesurable entre la cible au niveau du CL telle que déterminée selon l'atlas d'anatomie stéréotaxique/l'IRM et la localisation neurophysiologique par SCP.

Can J Neurol Sci. 2013; 40: 572-575

Medically refractory Parkinson's disease has been shown to benefit from deep brain stimulation (DBS). Stereotactic targets include nucleus ventralis intermedius, globus pallidus internus, and subthalamic nucleus (STN). Target choice depends on the primary clinical symptom of interest. Electrode implantation in STN is one of the more common locations, yielding benefit in rigidity, bradykinesia, and tremor, with reductions in oral medications ${ }^{1}$.

Classically, stereotactic localization of STN relies on an anatomical atlas to provide coordinates for frame based lead placement. Recent literature debates the application of 3 Tesla magnetic resonance imaging (MRI) with direct nucleus visualization in order to achieve proper STN localization ${ }^{2,3}$. Controversy surrounding MRI signal distortion and accurate nucleus delineation exists, though some use this as their primary modality for stereotactic coordinate acquisition for STN.
For those neurosurgeons who continue to utilize an anatomical atlas as a means to STN targeting, microelectrode recording (MER) has a controversial role to play ${ }^{4}$. The use of a microelectrode to record cellular activity can significantly aid in localization of nuclear groups and guide the surgeon to best final lead placement ${ }^{5}$. Brain shift, secondary to venting of spinal fluid through the operative site, can alter STN location from that predicted by atlas and pre-operative MRI. Thus, MER in the

From the Department of Surgery, Section of Neurosurgery, University of Manitoba, Winnipeg, Manitoba, Canada.

Received November 6, 2012. Final Revisions Submitted January 15, 2013. Correspondence to: Frederick A. Zeiler, Section of Neurosurgery, University of Manitoba, GB-1 820 Sherbrook Street, Health Sciences Center, Winnipeg, Manitoba, R3A 1R9, Canada. Email: umzeiler@cc.umanitoba.ca. 
setting of brain sag $^{6}$ carries the potential to aid in accurate localization of the STN. In addition, somatotopic organization of the STN can be elicited which may aid in symptom control ${ }^{7}$.

However, the need for intraoperative neurophysiological interpretation of the recording, cost of equipment, and prolongation of operative time raise concerns about cost effectiveness of MER as most final lead placements are close to predicted by anatomical stereotactic atlases ${ }^{8}$.

We retrospectively reviewed our intraoperative records for STN DBS in order to attempt to quantify the degree of discrepancy between predicted and MER guided final lead placement. In addition, we describe the significant variation in STN size and location compared to that predicted by stereotactic atlas.

\section{Methods}

A retrospective review of the operative reports of all patients receiving STN DBS implantation for Parkinson's disease at our institution from January 2004 to March 2011 was done.

Data on STN predicted and MER measured dorsal and ventral boundaries were recorded for both left and right sided trajectories. The number of tracts and tract position relative to the anatomical atlas pre-operatively planned trajectory was also recorded.

\section{RESULTS}

\section{Operative Technique}

By standard, we conduct MER for all STN DBS at our institution. Stereotactic coordinates of both the anterior commissure (AC) and posterior commissure (PC) were achieved via MRI with a Leksell stereotactic frame placed the morning of surgery. Using both AC/PC coordinates and the Shaltenbrand stereotactic anatomical atlas, the coordinates for STN target were determined. We used the Schaltenbrand atlas sagittal reconstruction maps at $12 \mathrm{~mm}$ lateral to $\mathrm{AC}-\mathrm{PC}$ midline for all STN cases. Target was chosen at STN center. Patients were operated on in the semi-Fowlers' position using the Leksell stereotactic frame and local nerve blocks. The patients were awake during the entirety of the MER and lead implantation. By convention, left side was operated on first.

Our technique involved parallel MER trajectories, with the criteria for entrance into the STN is a marked increase in background noise and the presence of multiple, high firing rate unit activity. High firing rate was defined as greater than 25-30 $\mathrm{Hz}$. The recordings indicating STN were typically characterized by irregular firing and/or bursting activity. Exiting the STN was characterized by an abrupt decrease in background noise and electrical silence. We do utilize intraoperative kinesthetic cellular response to aid in STN localization. We did not rely on somatopic delineation of STN to guide placement. Our MER defined target is usually a track containing greater than $4 \mathrm{~mm}$ of STN recording, plus a positive response to test stimulation. Additionally this track will have no side effects, or side effects evoked only by stimulation current of greater than $3 \mathrm{~mA}$.

Once these conditions are satisfied we usually position the distal contact at the MER-defined ventral border in order to have as many contacts as possible within the STN. We utilize Medtronic 3389 lead with four electrodes, each $1.5 \mathrm{~mm}$ in length, with $0.5 \mathrm{~mm}$ spacer.

\section{MER Tracts}

Within the time frame of the review, a total of 44 patients were operated on for bilateral STN DBS with intraoperative MER for Parkinson's disease. One patient was unable to receive final DBS lead placement due to poor localization of the STN with MER, and significant side effects with stimulation at predicted STN location. Thus, the total number of patients included in the review was 43 .

Table 1: STN Boundaries - Atlas predicted versus MER defined

\begin{tabular}{|c|c|c|c|}
\hline & $\begin{array}{c}\text { Average } \\
\text { Overall }\end{array}$ & $\begin{array}{l}\text { Average for } \\
\text { Right Side }\end{array}$ & $\begin{array}{l}\text { Average for } \\
\text { Left Side }\end{array}$ \\
\hline Dorsal Boundary STN - MER (mm above target) & 2.6 & 2.5 & 2.6 \\
\hline Dorsal Boundary STN - Atlas predicted (mm above target) & 5.0 & N/A & N/A \\
\hline Ventral Boundary STN - MER (mm below target) & 2.0 & 1.8 & 2.2 \\
\hline Ventral Boundary STN - Atlas predicted (mm below target) & 1.4 & N/A & N/A \\
\hline DV-Distance STN - MER (mm) & 4.6 & 4.3 & 4.8 \\
\hline DV-Distance STN - Atlas (mm) & 6.4 & N/A & N/A \\
\hline
\end{tabular}

$\mathrm{STN}=$ subthalamic nucleus, $\mathrm{MER}=$ microelectrode recording, $\mathrm{mm}=$ millimetre $, \mathrm{DV}=\mathrm{dorsal} / \mathrm{ventral}, \mathrm{N} / \mathrm{A}=$ not applicable . 
Table 2: Differences in STN Measurements between MER and Atlas Predicted

\begin{tabular}{|c|c|c|c|}
\hline & $\begin{array}{l}\text { Avg Difference in } \\
\text { Dorsal Boundary }\end{array}$ & $\begin{array}{l}\text { Avg Difference in } \\
\text { Ventral Boundary }\end{array}$ & $\begin{array}{l}\text { Avg Difference in DV- } \\
\text { distance }\end{array}$ \\
\hline MER vs. Atlas (mm) & 2.4 (deeper) & 0.6 (deeper) & 1.9 (smaller) \\
\hline
\end{tabular}

A total of 209 tracts with MER were mapped, with an average of 2.4 per side (range: $1-11$ ). The average number of tracts on the left was 2.3 (range: $1-5$ ), while the average number of tracts on the right was 2.4 (range: $1-11$ ).

\section{STN Boundaries: Atlas Predicted vs. MER defined}

The dorsal STN boundary as predicted by the atlas was on average $5.0 \mathrm{~mm}$ above the target (designated as $-5.0 \mathrm{~mm}$ ). Using MER, the dorsal boundary of STN was $2.6 \mathrm{~mm}$ above atlas predicted target on average (range: $0.5 \mathrm{~mm}$ above target to $7.0 \mathrm{~mm}$ above target). The dorsal boundary of STN using MER for the right and left side was $2.5 \mathrm{~mm}$ and $2.6 \mathrm{~mm}$ above predicted target respectively.

Similarly, the ventral boundary of STN, as predicted by atlas, was on average $1.4 \mathrm{~mm}$ below target. With MER, the ventral boundary of STN was $2.0 \mathrm{~mm}$ below target (range: $2 \mathrm{~mm}$ above target to $6 \mathrm{~mm}$ below target). Ventral boundary of STN using MER for the right and left side were $1.8 \mathrm{~mm}$ and $2.2 \mathrm{~mm}$ below target on average respectively.

Overall, the dorsal-ventral (DV) length of STN as predicted by atlas was $6.4 \mathrm{~mm}$. As per MER, the average DV distance was $4.6 \mathrm{~mm}$ (range: $2-7.5 \mathrm{~mm}$ ). The DV distance of the right and left STN was determined to be $4.3 \mathrm{~mm}$ and $4.8 \mathrm{~mm}$ on average, respectively.

To summarize, the dorsal boundary of STN was determined to be $2.4 \mathrm{~mm}$ deeper as per MER compared to predicted atlas location. In addition, the ventral boundary of STN was displayed to be $0.6 \mathrm{~mm}$ deeper as per MER compared to predicted atlas location. The DV distance of STN was demonstrated to be 1.9 $\mathrm{mm}$ smaller than predicted by stereotactic atlas. Finally, the STN center was located on average $2 \mathrm{~mm}$ deeper as per MER, compared to atlas. Summary of atlas and MER STN boundaries and DV distances can be seen in Table 1. Overall differences between STN boundaries between atlas and MER are displayed in Table 2.

\section{Final Lead Position}

Final lead position was defined in reference to distal contact placement relative to predicted atlas target. Average change in final lead placement was $1.5 \mathrm{~mm}$ deeper than predicted by atlas in the dorsal-ventral axis, guided by MER (range: $2.5 \mathrm{~mm}$ above predicted target to $5.5 \mathrm{~mm}$ below predicted target).
In 19 patients, final lead placement was $2.6 \mathrm{~mm}$ anterior to that predicted by atlas, while nine patients had final lead placement $2 \mathrm{~mm}$ medial to the predicted target.

All alterations in final lead position from predicted atlas target produced clinically significant reduction in stimulation side effects intra-operatively. There were no clinically significant side effects as a result of MER, including hemorrhages.

\section{DisCUSSION}

Controversy over MER in STN DBS exists, related to a variety of reasons. First, concerns regarding increased complication rates secondary to multiple passes during MER, in attempt at optimal DBS lead placement, have been raised. Gorgulho et $\mathrm{al}^{9}$ attempted to quantify the theoretical incidence of increased hemorrhage ${ }^{10}$. In retrospective analysis of 178 patients identified to have undergone DBS, the overall incidence of hemorrhage was $2.9 \%$ in those with MER versus $1.4 \%$ without MER being used. However, this failed to display statistical significance. In addition, the clinical significance of these hemorrhages was unknown. Finally the rate of hemorrhage was statistically associated with pre-existing hypertension as a medical co-morbidity.

Second, a US based cost analysis of DBS with and without the use of MER demonstrated an increased cost for unilateral and bilateral cases of $\$ 19,461.75$ and $\$ 20,525.98$ respectively ${ }^{8}$. These costs are cumulative sums based on equipment, personnel, operative room time and anesthesia costs for increased duration of DBS cases related to MER use. One could argue however, that these costs may be offset by less need for repeat clinical followup for stimulator programming and medication alterations due to sub-optimal lead placement in the absence of MER during surgery.

Finally, some argue that regardless of accuracy of lead placement, STN lead placement can occur within a $6 \mathrm{~mm}$ cylinder of tissue at presumed STN center with no clinical difference in outcome ${ }^{11}$. This has yet to be confirmed in the literature.

The benefits of MER are fairly clear. It allows for somatotopic delineation within the $\mathrm{STN}^{7}$. The intra-operative neurophysiology that is displayed with MER offers STN topographic information ${ }^{12}$ with characteristic neuronal activity that can confirm one's assumption of entering and exiting the 
nuclear group and surrounding structures ${ }^{13,14}$. Finally, arguably the most important factor, is that MER can alter final lead placement. Recent literature by Senatus et $\mathrm{al}^{4}$ displays the variation in dorsal-ventral STN center, indicating it being more deeply located compared to the position predicted by anatomical atlas. The significance of this variation in lead placement to clinical outcome has yet to be determined.

Our data provides consistent results to those previously described $^{4}$. The use of MER in our series found the overall STN center to be $2 \mathrm{~mm}$ deeper compared to atlas. No difference occurred between left to right during the operation. Thus, this discrepancy is suspected to be due to brain shift after the initial dural opening. The interesting finding is that the brain shift does not seem to worsen after moving from left to the right side for lead placement. Our data also suggests that the overall STN size in DV-distance is smaller than predicted by the stereotactic atlas by $1.9 \mathrm{~mm}$ on average (Table 2). This is important as the average DV-distance was only $4.6 \mathrm{~mm}$, meaning a placement of the final lead "anywhere" within a $6 \mathrm{~mm}$ cylinder of STN predicted center ${ }^{11}$, could definitely produce lead placement outside of the nuclear group. Overall, our final lead placement was changed by $1.5 \mathrm{~mm}$ deeper than predicted by atlas, in the dorsal-ventral axis of STN. Even though a small distance ${ }^{4}$, this can change the number of lead contacts located within the STN, and thus significantly alter the available electrode configurations and stimulation parameters post-operatively. This could potentially mean the difference between good and excellent clinical response to stimulation. Thus, our preference and recommendation is to utilize MER when conducting STN DBS.

Despite our results in favor of MER for STN DBS, we recognize there are limitations to the conclusions that can be made. First, the study is retrospective in nature, and the topic in question would benefit from a prospective analysis comparing MER to atlas targeting. Second, our numbers are small, making the results difficult to generalize to STN DBS in all institutions. Third, long-term clinical outcome data on final stimulation parameters and electrode contact configuration have yet to be analyzed. Comparing these to final lead placements may shed light on whether or not vigilance with MER guided DBS placement truly impacts clinical outcome. Furthermore, we lack intra-operative objective clinical assessment during stimulation at atlas predicted versus MER defined targets, which may provide useful additional information. Finally, a randomized prospective study comparing intra-operative DBS results with and without intra-operative STN MER may be worth consideration.

\section{Conclusions}

Microelectrode recording in STN DBS surgery demonstrated measurable difference between stereotactic atlas STN target and neurophysiologic STN localization. Furthermore, a large proportion of trajectories were moved anterior as a result of atlas inaccuracies. This technique provided invaluable targeting information, allowing for accurate delineation of STN borders and confidence in final electrode placement.

\section{REFERENCES}

1. Deep-brain Stimulation for Parkinson's Disease Study Group. Deep-brain stimulation of the subthalamic nucleus or the pars interna of the globus pallidus in Parkinson's disease. N Engl J Med. 2001;345(13):956-63.

2. Foltynie T, Zrinzo L, Martinez-Torres, et al. MRI-guided STN DBS in Parkinson's disease without microelectrode recording: efficacy and safety. J Neurol Neurosurg Psychiatry. 2011;82: 358-63.

3. Abosch A, Yacoub E, Ugurbil K, et al. An assessment of current brain targets for deep brain stimulation surgery with susceptibility-weighted imaging at 7 Tesla. Neurosurg. 2010;67 (6):1745-56.

4. Senatus PB, Teeple D, McClelland S, et al. A technique for minimally altering anatomically based subthalamic electrode targeting by microelectrode recording. Neurosurg Focus. 2006; 20(5):E8

5. Chen SY, Lee CC, Lin SH, et al. Microelectrode recording can be a good adjunct in magnetic resonance image-directed subthalamic nucleus deep brain stimulation for parkinsonism. Surg Neurol. 2006;65:253-61.

6. Obuchi T, Katayama Y, Kobayashi K, et al. Direction and predictive factors for the shift of brain structure during deep brain stimulation electrode implantation for advanced Parkinson's disease. Neuromodulation. 2008;11(4):302-10.

7. Romanelli P, Heit G, Hill BC, et al. Microelectrode recording revealing a somatotopic body map in the subthalamic nucleus in humans with Parkinson disease. J Neurosurg. 2004;100:611-8.

8. McClelland S. A cost analysis of intraoperative microelectrode recording during subthalamic stimulation for Parkinson's disease. Mov Disord. 2011;26(8):1422-7.

9. Gorgulho A, De Salles AAF, Frighetto L, et al. Incidence of hemorrhage associated with electrophysiological studies performed using macroelectrodes and microelectrodes in functional neurosurgery. J Neurosurg. 2005;102:888-96.

10. Hariz MI. Surgical probing into the basal ganglia: hemorrhage and hardware-related risks, and costs of microelectrode recording. Mov Disord. 2011;26(8):1375-7.

11. McClelland S, Ford B, Senatus PB, et al. Subthalamic stimulation for Parkison disease: determination of electrode location necessary for clinical efficacy. Neurosurg Focus. 2005;19 (5):E12.

12. Seifried C, Weise L, Hartmann R, et al. Intraoperative microelectrode recording for the delineation of subthalamic nucleus topography in Parkinson's disease. Brain Stim. 2012;5 (3):378-87.

13. Hutchison WD, Allan RJ, Opitz H, et al. Neurophysiological identification of the subthalamic nucleus in surgery for Parkinson's disease. Ann Neurol. 1998;44(4):622-8.

14. Sterio D, Zonenshayn M, Mogilner AY, et al. Neurophysiological refinement of subthalamic nucleus targeting. Neurosurg. 2002; 50(1):58-69. 